\title{
Morphological Study of the Golgi Tendon Organ in Equine Superficial Digital Flexor Tendon
}

\author{
By

\begin{abstract}
Takafumi WATANABE ${ }^{1}$, Yoshinao HOSAKA ${ }^{1}$, Etsuko YAMAMOTO ${ }^{1}$,
\end{abstract} \\ Hiromi UEDA ${ }^{1}$, Prasarn TANGKAWATTANA ${ }^{2}$ and Kazushige TAKEHANA ${ }^{1}$ \\ ${ }^{1}$ Department of Veterinary Anatomy, Rakuno Gakuen University, Ebetsu, Hokkaido 069-8501, Japan \\ ${ }^{2}$ Department of Veterinary Anatomy, Khon Kaen University, Khon Kaen 40002, Thailand
}

- Received for Publication, April 19, $2004-$

\begin{abstract}
Key Words: Golgi tendon organ, Locomotorium, nerve, superficial digital flexor tendon
Summary: The Golgi tendon organ (GTO) is an encapsulated fusiform mechanoreceptor siding in the musculotendinous junction of many animal species. Inhibitory function of afferent nerve fibers distributed from nearby motor units, the organ responds to active tension exerted onto the muscle. The morphological features of the equine GTO have not yet been elucidated. Additionally, there is some controversy regarding to the existence of the GTO in the equine superficial digital flexor tendon (SDFT). Therefore, immunohistochemistry and immunoelectron microscopy using alcian blue ( $\mathrm{pH}$ 2.5) staining and the silver-enhanced colloidal gold method were carried out to determine both the location and characteristics of the GTO at the musculo-tendinous junction of the SDFT. A GTO with a fusiform structure of approximately $3 \mathrm{~mm}$ in length was found in the tendinous part. The lumen of the GTO was divided into compartments by septal cells. Each compartment contained collagen fibrils, nerve fibers and Schwann cells. This is the first report of the equine GTO.
\end{abstract}

The Golgi tendon organ (GTO) is a mechanoreceptor that is generally located along musculotendinous and musculo-aponeurotic boundaries in various animals ${ }^{1}$. The mechanoreceptor is sensitive to mechanical exertion during passive muscle stretching or active muscle contraction involved in the regulation of motor reflex. This wholly encapsulated structure is innervated by one or two afferent nerve fibers of large diameters from a nearby motor unit ${ }^{2,3)}$. Generally, the receptor has four axonal profiles: a large myelinated axon (type I), an unmyelinated axon surrounded by Schwann cell processes and a basal lamina (type II), an unmyelinated axon covered only by a basal lamina (type III) and an unmyelinated axon not covered either by a Schwann cell layer or a basal lamina (type IV).

The type IV unmyelinated axon therefore has its axolemma located close to the surrounding collagen bundle. The capsule lumen inside each GTO is divided into irregular longitudinal compartments by delicate processes of septal cells. The collagen bundles divide into sub-bundles intertwining with unmyelinated axons, and each subunit spirals down along the longitudinal axis of the capsule lumen for a short distance before reunion ${ }^{1)}$. Contraction of skeletal muscle and stretching of collagen fibrils in the GTO capsule lumen, evoke the release of inhibitory postsynaptic potential from intertwining $\mathrm{Ib}$ axons $^{4-9)}$.

Superficial digital flexor tendonitis frequently occurs in the forelimbs of Thoroughbred horses during racing work ${ }^{10)}$. Although the GTO is known to be an important organ for exercise control, its structure in horses has not been clarified. It has been reported that a GTO is not present in the superficial digital flexor tendon ${ }^{11)}$ and that a lumbrical muscle exists between the SDFT and the deep digital flexor tendon of horses ${ }^{12)}$. This would lead to an incidental number of SDFT inflammations. Therefore, the aim of the present study was to verify the presence of the GTO and to determine the characteristics of the GTO in the equine SDFT. 


\section{Materials and Methods}

Thirteen normal musculo-tendinous tissues removed from SDFTs of 7 male and 6 female Thoroughbred horses (aged from newborn to 18 years) were used in this study. The horses were housed at the Department of Veterinary Pathology in Rakuno Gakuen University, Hokkaido, Japan. After intramuscular sedation with medetomidine hydrochloride $(16 \mathrm{mg} / \mathrm{kg})$ induction of anesthesia with thiopental sodium (6 mg/kg, IV), approximately 20mm-long tissue samples were surgically removed. All animal experiments were approved by the Ethics Committee and the Institutional Animal Use and Care Committee of Rakuno Gakuen University. The samples were fixed in $4 \%(\mathrm{w} / \mathrm{v})$ paraformaldehyde in $0.1 \mathrm{M}$ sodium phosphate buffer ( $\mathrm{pH} 7.4$ ) for $6 \mathrm{hr}$, immersed in $30 \%(\mathrm{w} / \mathrm{v})$ sucrose solution ( $\mathrm{pH} 7.4)$ overnight at $4^{\circ} \mathrm{C}$, embedded in Tissue-Tek O.C.T compound (Sakura Finetechnical Co., Ltd. Tokyo, Japan), and then frozen in liquid nitrogen. The frozen samples were sectioned longitudinally at a thickneses of $20 \mu \mathrm{m}$ for both light and immunoelectron microscopic studies.

\section{Light microscopic observation}

The sections were mounted on poly-L-lysinecoated glass slides (Polysciences, Warrington, PA, USA). Acidic mucosubstance localized between collagen fibrils in the GTO were identified by staining with alcian blue $(\mathrm{pH} 2.5)$. Counter staining with nuclear fast red to identify the nucleus was also performed. Nerve fibers terminating and distributed in the GTO were immunohistochemically examined by the silver-enhanced colloidal gold method using anti-Protein gene product 9.5 (PGP 9.5) serum. The sections were serially processed by pre-treatment with normal goat serum, incubation with rabbit anti-human PGP 9.5 serum (1:7,000, Ultraclone, Cambridge, UK), incubation with a colloidal gold-conjugated anti-rabbit IgG (Nanogold; $1.4 \mathrm{~nm}$, Nanoprobes, Yaphank, NY, USA) diluted 1:30 with $0.1 \%(\mathrm{w} / \mathrm{v})$ bovine serum albumin-phosphate buffer $(\mathrm{pH} 7.2)$ overnight at $4{ }^{\circ} \mathrm{C}$, fixation with $3 \%(\mathrm{v} / \mathrm{v})$ glutaraldehyde for 10 min, and immersion in $0.1 \mathrm{M}$ acetic acid buffer $(\mathrm{pH}$ 7.0) for $10 \mathrm{~min}$ to yield detailed electron immunoreactive structures. Silver enhancement was performed by the use of a silver enhancement kit (HQ Silver; Nanaoprobes) in a dark room for $8 \mathrm{~min}$.

\section{Immunoelectron microscopic observation}

The samples that showed positive reaction to immunostaining were postfixed in $1 \%(\mathrm{w} / \mathrm{v}) \mathrm{OsO}_{4}$ for $30 \mathrm{~min}$, dehydrated through a graded series of ethanol, and embedded in Quetol 812 (Nisshin EM,
Tokyo, Japan) according to the standard procedure. Ultrathin sections were cut on an ultramicrotome (Reichert Supernova system; Leica, Vienna, Austria), mounted on copper grids, stained with $1 \%(\mathrm{w} / \mathrm{v})$ uranyl acetate and $2 \%(\mathrm{w} / \mathrm{v})$ lead citrate, and examined through a transmission electron microscope (JEM-1220; JEOL, Tokyo, Japan). Some sections were additionally stained with $0.2 \%$ (w/v) tannic acid solution (Merck, Tokyo, Japan) to obtain more electron microscopic information.

\section{Results}

\section{Light microscopic observation}

Muscle fibers diagonally inserted into collagen fibrils were found in the musculo-tendinous junction. Fusiform structures, approximately $3 \mathrm{~mm}$ long and $0.3 \mathrm{~mm}$ wide, were found between collagen fibrils and muscle fibers and the structures coursed parallel with the longitudinal axis of collagen fibrils of the tendon. (Fig. 1). These structures showed positive reaction to alcian blue staining and also had circular and large-sized nuclei, thought to be Schwann cells, in its lumen (Fig. 2).

\section{Electron microscopic observation}

In the immunoreactivity area, each fusiform structure was divided into compartments by processes of large non-lamella cells. Each compart-

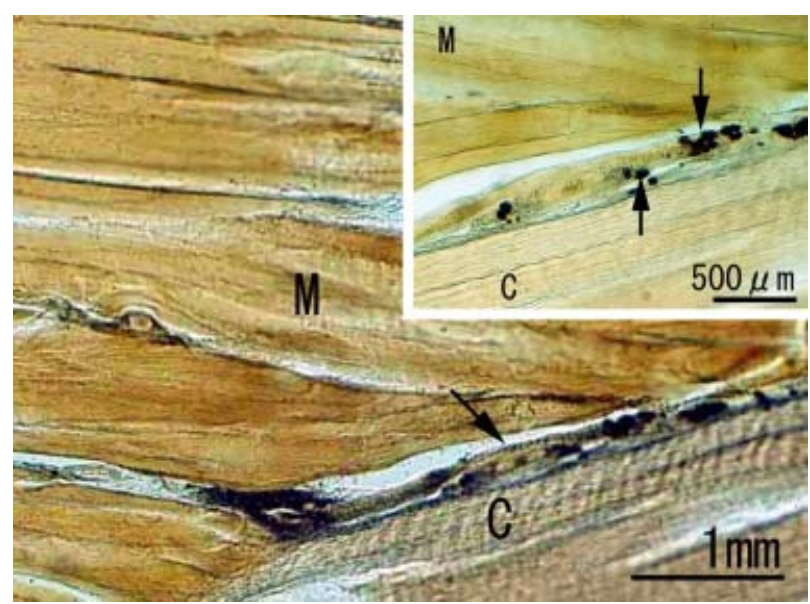

Fig. 1. Musculo-tendinous junction of the superficial digital flexor tendon on a frozen section preparation stained with PGP 9.5. Golgi tendon organ (GTO) is (arrow) coursing in parallel with the longitudinal axis of the tendon. Insert: GTO is made a fusiform structure in the tendinous parts. The structure yields a strong positive silver-enhanced colloidal gold reaction (arrows). C: superficial digital flexor tendon. M: superficial digital flexor muscle. 


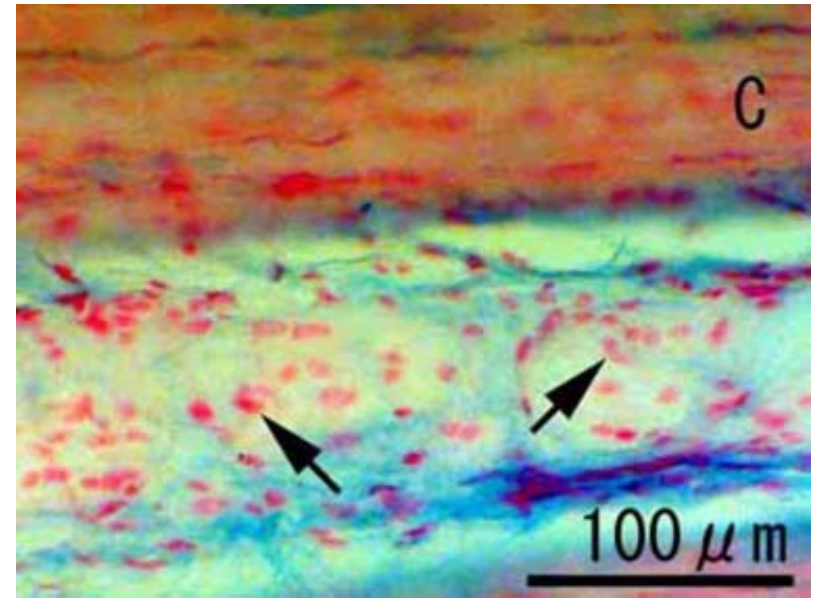

Fig. 2. Musculo-tendinous junction of the SDFT (C). Alcian blue $(\mathrm{pH} 2.5)$ reacts positively with the fusiform structure running parallel to the collagen bundle. This structure has many circular and large-sized nuclei (arrows).

ment was occupied by collagen fibrils intertwining with axonal fibers (Fig. 3). Coursing patterns of collagen fibrils observed in each section varied from longitudinal, transverse to oblique (Fig. 4). In this study, axonal profiles could be categorized into three types: (1) unmyelinated axons surrounded by Schwann cell processes and basal lamina (Fig. 5 a), (2) unmyelinated axons covered by a basal lamina (Fig. 5 b) and (3) unmyelinated axons not covered by either a Schwann cell layer or a basal lamina. Because of the luck of Schwann cells and basal

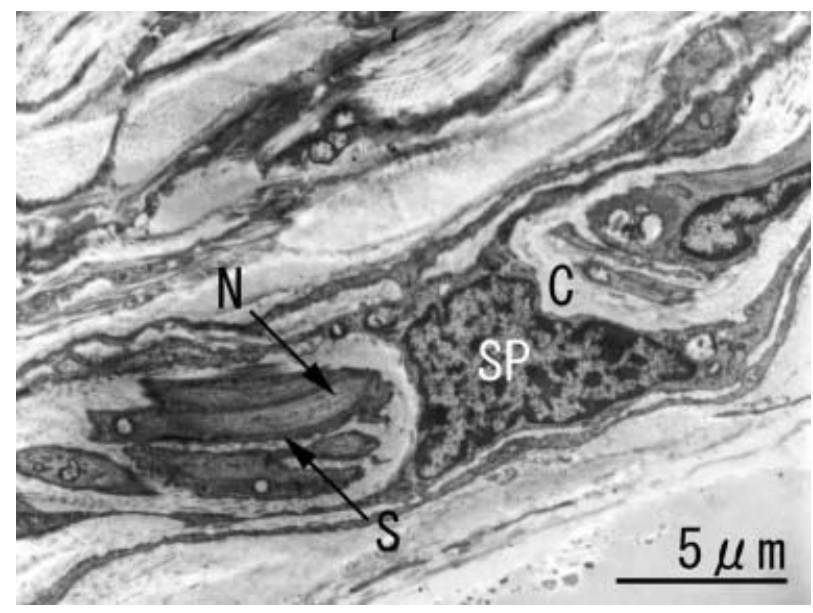

Fig. 3. The processes of septal cell (SP) divided the fusiform structure into compartments. C: collagen fibrils, $\mathrm{N}+$ arrow: nerve fiver, $\mathrm{S}+$ arrow: Schwann cell processes surrounding the nerve fiber.

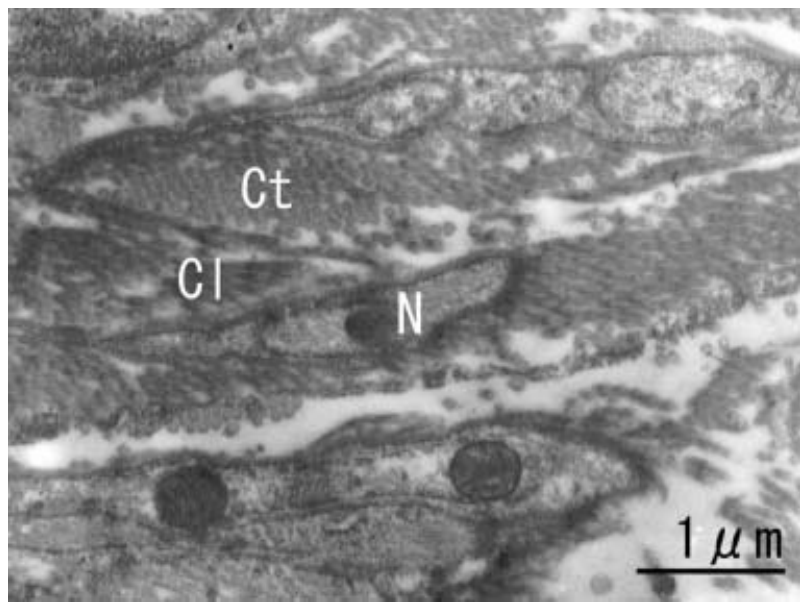

Fig. 4. Collagen fibrils stained with tannic acid. In each compartment, the nerve fibers $(\mathrm{N})$ are surrounded by collagen fibrils with both transverse $(\mathrm{Ct})$ and longitudinal $(\mathrm{Cl})$ orientations.

lamina in the third profile, axolemma of each axon in this profile was brought to place closely against its surrounding collagen bundle (Fig. 5 c). Nerves in the equine GTO could be classified into types II, III, and IV in accordance with results reported by Schoultz and Swett ${ }^{1}$. Several nerves were found to possess indentations on their surfaces (Fig. 6). These indentations appear to be sites for insertion of transversely oriented collagen fibrils.

\section{Discussion}

Many nerve fibers surrounded by circular and large-sized nuclei were distributed in the fusiform structure. The nuclei of cell stained with nuclear fast red were thought to be Schwann cells. Type I nerve fibers are restricted to the region where $\mathrm{Ib}$ afferent nerve fibers enter the GTO in other animals, but this nerve fiber type was not found in this study. To confirm this finding, complete serial sections of the equine GTO should be examined.

Coursing patterns of collagen fibrils observed in planes of a section were longitudinal, transverse and oblique. In contrast, collagen fibrils in the equine GTO coursed spirally down along the longitudinal axis of the capsule lumen, in accordance with results reported ${ }^{1)}$. The helical constitution of the GTO collagen fibrils also positively and strongly reacted with the antibodies against acidic mucopolysaccharides of glycosaminoglycans ${ }^{3,13}$. These findings indicate that the extracellular matrix of equine GTO contains compact collagen fibrils 

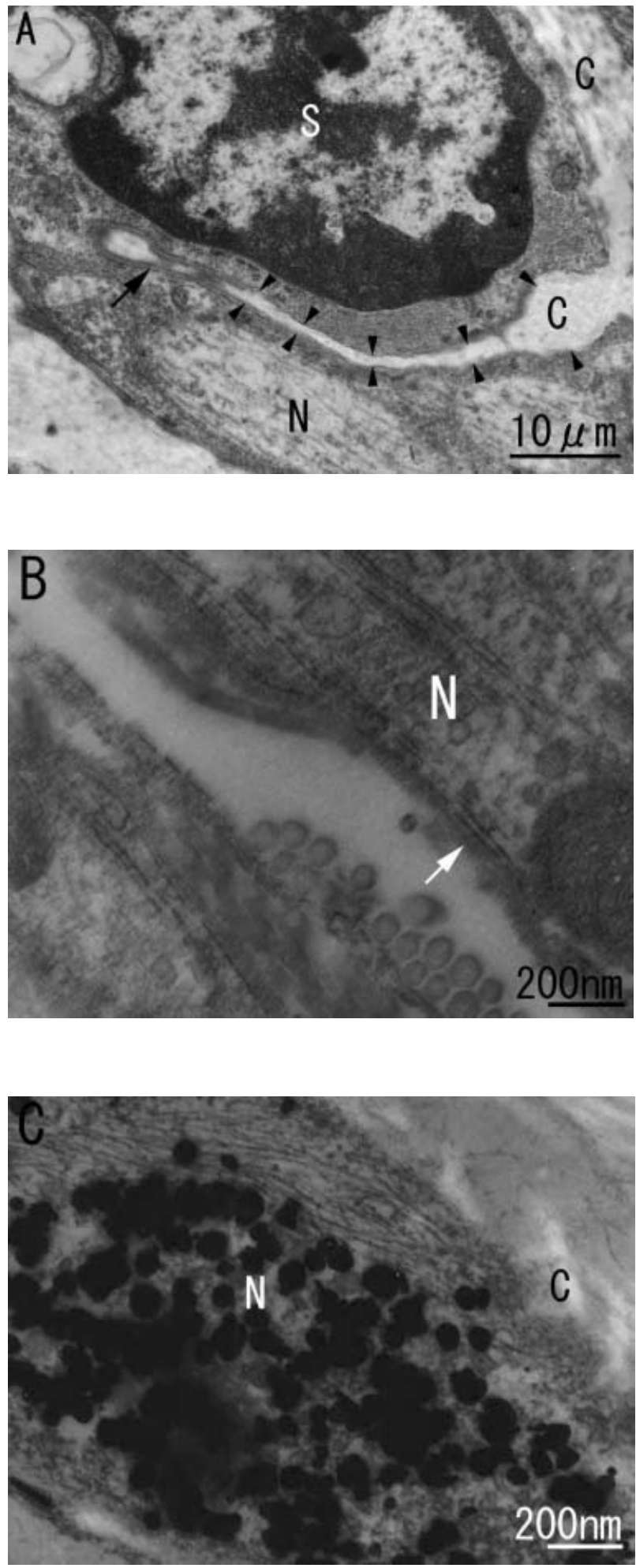

Figs. 5. a: Nerve fiber (N) surrounded by Schwann cell processes (S) and a basal lamina (arrow). Schwann cell processes wrapped around the collagen fibrils (C) (arrowheads). b: Tannic acid staining. Nerve fiber covered by a basal lamina (white arrow). c: Bare nerve fiber in direct contact with collagen fibrils (C). Nerve fiber reacted specifically to PGP 9.5.

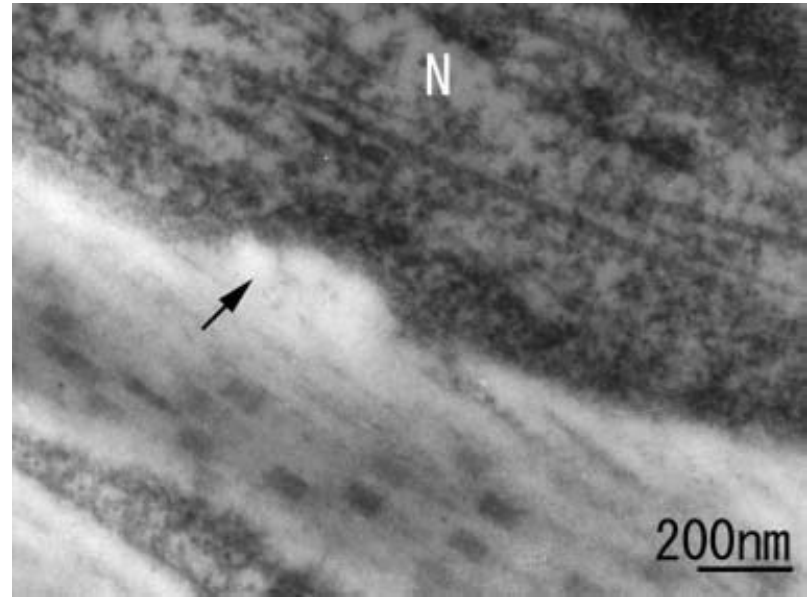

Fig. 6. Cross-sectional collagen fibrils (arrow) can be seen at the indentation of a nerve fiber $(\mathrm{N})$.

and high contents of acidic mucopolysaccharides in glycosaminoglycans.

The fusiform structure was divided into compartments by the processes of large non-lamella cells. Nerve fibers in each compartment were surrounded by collagen fibrils. Septal cells without basal lamina that appeared in the GTO could be set into the receptor ${ }^{1}$.

Since the above-described features of the equine GTO are in agreement with those reported in the musculo-tendinous junction of the other animals except for the type I nerve fibers ${ }^{1,3,4,8}$. It can be concluded that the GTO exists in the superficial digital flexor tendon of horses.

Collagen fibrils come into contact with indentations, known as caveolae, on Schwann cell projections in afferent nerve fibers ${ }^{14}$. In addition, collagen fibrils are also inserted into rugged areas on distal parts of the nerve fibers. It was seemingly that the nerve fibers increased contacting areas for collagen fibrils. Generally, once a nerve impulse has arrived at a sensory receptor, it is translated to a signal evoking action potential on the afferent nerve. Strength of the action potential has been shown to be proportionate to the strength of the signal. Proportions of the strength of stimulating signals on the receptors and of the action potential on the neurolemma of the afferent nerve are known as frequency encoding ${ }^{5)}$.

The contact between nerve fibers and collagen fibrils in the GTO observed in this study suggest to provide afferent nerve fibers with more detailed signals from muscle contraction. 


\section{References}

1) Shoultz TW and Swett JE. The fine structure of Golgi tendon organ. J Neurocytol 1972; 1:1-26.

2) Barker D. The structure and distribution of muscle receptors. In: Barker D ed. Symposium on Muscle Receptors. Hong Kong. Hong Kong University Press. 1962; $227-$ 240.

3) Blumer R, Lukas RJ, Wasicky R and Mayr R. Presence and morphorogical variability of Golgi tendon organs in the distal portion of sheep extraocular muscle. Anat Rec 2000; 258:359-368.

4) Bridgman CF. The structure of tendon organs in cat: a proposed mechanism for responding to muscle tension. Anat Rec 1968; 162:209-220.

5) Cunningham GJ. Skeletal muscle strech receptors. In: Cunningham GJ ed. Textbook of Veterinary Physiology. Philadelphia. B. W. Saunders. 1992; 57-63.

6) Danie MK, Joshua TT and Wesley JT. Glial growth factor rescues Schwann cells of mechanoreceptors from denervation-induced apoptosis. J Neurosci 1997; 17:66976706.

7) Keele AC and Neil E. Reflex action. In: Keele AC and Neil E eds. Samson Wright's Applied Physiology, 12th ed. London. Oxford University Press. 1971; 280.
8) Ruskell GL. The incidence and variety of Golgi tendon organs in extraocular muscles of the rhesus monkey. $J$ Neurocytol 1979; 8:639-653.

9) Wohlfart $G$ and Henriksson GK. Observations on the distribution, number and innervation of Golgi musculetendinous organs. Acta Anat 1960; 41:192-204.

10) Anesaki R. Disease of musculature. In: Equine Research Institute Japan Racing Association ed. Equine Veterinary Medicine, 1st ed. Tokyo. Chikusan Shuppansha. 1996; 293 243 (in Japanese).

11) Yamaguchi M. Education system and research of equine muscule-tendinous junction. Equine Science 2001; 37:363364 (in Japanese).

12) Klomkleaw K, Kasashima Y, Fuller AG, Kobayshi A, Yoshihara T, Oikawa MA, Izumisawa Y and Yamaguchi M. Horse lumbrical muscle: possible structural and functional reorganization in regressive muscle. Anat Histol Embryol 2002; 31:85-98.

13) Pearse AGE. In: Pearse AGE ed. Histochemistry, Theoretical and Applied, 4th ed. Edinburgh. Analytical Technology Livingstone. 1985; 2:578-754.

14) Maeda $T$, Sato $O$, Kobayashi $S$, Iwanaga $T$ and Fujita $T$. The ultrastructure of Ruffini endings in the peridontal ligament of rat incisors with speceal reference to the terminal Schwann cells (K-Cell). Anat Rec 1989; 223:95-103. 